Research Article

\title{
Multiple Attribute Group Decision Making Based on Simplified Neutrosophic Integrated Weighted Distance Measure and Entropy Method
}

\author{
Haibo Zhang $\mathbb{D}^{1}{ }^{1}$ Zhimin $\mathrm{Mu}^{2}$ and Shouzhen Zeng $\mathbb{D D}^{1}$ \\ ${ }^{1}$ School of Business, Ningbo University, Ningbo 315211, China \\ ${ }^{2}$ Department of Basic Science, Tianjin Agricultural University, Tianjin 300384, China \\ Correspondence should be addressed to Haibo Zhang; haibo12500@163.com and Shouzhen Zeng; zszzxl@163.com
}

Received 2 June 2020; Accepted 18 June 2020; Published 20 July 2020

Guest Editor: Zaoli Yang

Copyright (C) 2020 Haibo Zhang et al. This is an open access article distributed under the Creative Commons Attribution License, which permits unrestricted use, distribution, and reproduction in any medium, provided the original work is properly cited.

Simplified neutrosophic set (SNS) is a popular tool in modelling potential, imprecise, and uncertain information within complex environments. In this paper, a method based on the integrated weighted distance measure and entropy weight is proposed for handling SNS multiple attribute group decision-making (MAGDM) problems. To this end, the simplified neutrosophic (SN) integrated weighted distance (SVNIWD) measure is first developed for overcoming the limitations of the existing methods. Afterward, the proposed SNIWD's several properties and particular status are studied. Moreover, a flexible and useful MAGDM approach that combines the strengths of the SNIWD and the SNS is proposed, wherein the SN entropy measure is applied to calculate the unknown weight information regarding attributes. Finally, a numerical case of investment evaluation and subsequent comparative analysis are conducted to prove the superiority of the proposed framework.

\section{Introduction}

The aim of the multiple attribute group decision-making (MAGDM) problem is to determine suitable alternatives with respect to multiple attributes according to the judgement provided by various decision makers. It is impossible for a decision maker to always express an accurate preference because of the increasing uncertainties of the assessed problems. To solve the difficulties, many effective mathematical tools are introduced during the decision process. The fuzzy set (FS) firstly developed by Zadeh [1] is widely used to model imprecise and vague information in MAGDM. An element's membership value in fuzzy theory lies in the range $[0,1]$, while the value of its complement is called the nonmembership. To provide a more effective method, the conception of intuitionistic FS (IFS) was proposed by Atanassov [2] which is described by membership and nonmembership functions, and their sum cannot exceed 1. Later, Yager [3] presented the Pythagorean FS (PFS), whose special merit is that the square sum of the membership and nonmembership shall lie in interval [01,]. Thus, the PFS is a more powerful tool to describe uncertainties than the IFS and FS. Up to now, the PFS has gained more and more attention and has been widely used in decision making as well as other areas [4-15].

Recently, Smarandache [16] defined the idea of the neutrosophic set (NS) utilizing three parameters: the degrees of truth, indeterminacy, and false for the first time. These three components in the NS are entirely irrelevant from each other, which help people present their preference more flexibly and accurately compared with the previous IFS and PFS. To enhance the computational efficiency of the NS, Wang et al. [17] and Ye [18] put forward the concept of simplified neutrosophic set (SNS). The SNS has gained increasing attention from researchers in these years because of its preponderance in describing uncertainties. For example, Ye [19] extended the TOPSIS method to handle simplified neutrosophic (SN) environments and studied its application in selecting suppliers. Peng et al. [20] introduced an outranking method for SN MAGDM problems. Peng et al. [21] 
developed some aggregation methods for SN information. Kucuk and Sahin [22] provided a hybrid method for SN decision-making in which the weight information is unknown. Ye [23] gave a netting approach to cluster SN information based on new associated coefficients. Sahin and Liu [24] developed several SN aggregation operators utilizing the possibility information. Liu and Luo [25] proposed a power aggregation to infuse the SNS and explored its usefulness in MAGDM. Ye [26] introduced a generalized ordered weighted SN cosine similarity measure and applied it to solve MAGDM problems. Zeng et al. [27] presented a novel TOPSIS approach for SN decision-making considering the high-efficiency correlation coefficient. Peng and Dai [28] conducted a bibliometric survey of the development concerning the neutrosophic set from 1998 to 2017.

Various distance measures have been put forward and used in decision-making process for reflecting the deviations between the arguments. One of the most widely used distances is the weighted distance, including weighted Hamming and weighted Euclidean distances [29]. Motivated by the ideal of the ordered weighted method [30], Xu and Chen [31] presented the ordered weighted distance (OWD) measure considering the importance of the ordered deviations by designing weight scheme. Later, Merigó and GilLafuente [32] presented the ordered weighted averaging distance (OWAD) measure, and applied it to evaluate financial products. So far, a variety extensions of the OWD and the OWAD measures have been presented in the literature. Xu and Xia [33] explored the OWD with hesitant fuzzy information and developed the hesitant fuzzy OWD and hybrid weighted similarity measures. Zeng and Su [34] adapted the OWD into the IFS situation and presented the intuitionistic fuzzy OWD (IFOWD). Zeng [37] studied the usefulness of the IFOWD using a generalized mean method. Shakeel et al. [38] developed the cubic OWD (COWD) and gave its application in decision-making. Zhou et al. [37, 38] worked on several continuous OWD measures. Some authors also extended the OWD and the OWAD using more complex variables, such as the logarithmic means $[39,40]$, induced aggregation [41-43], and weighted average [44-46]. More recently, considering the usefulness of the SNS, Sahin and Kucuk [47] proposed the simplified neutrosophic OWD (SNOWD) and studied its performance in the group decision-making problem.

With awareness the capabilities of the SNS which are analyzed above, the purpose of this research is to propose a new SN distance measure that can correct the shortcomings of the existing methods and apply it to MAGDM. To this end, we present the simplified neutrosophic integrated weighted distance (SNIWD) measure, which combines the significance of the existing SNOWD and SN weighted distance (SNWD) measures. Therefore, it can eliminate the limitations of the SNOWD that cannot account for the importance of attribute in MAGDM problems. Moreover, it generalizes a wide kind of existing SN distance measures, including the SNOWD and the SNWD measures. We also verify the merits of the proposed SNIWD measure by exploring its application to MAGDM problems, in which the weight information of attributes is unknown.
The reminder of this paper is carried out as follows: Section 2 gives the backgrounds of the SNS and the OWD measure. Section 3 defines the SNIWD measure and studies its main properties and various cases. Section 4 constructs a MAGDM model based on the SNIWD measure and entropy measure, and a mathematical example is provided in Section 5. Finally, Section 6 draws some valuable conclusions.

\section{Preliminaries}

\subsection{The Simplified Neutrosophic Set (SNS)}

Definition 1 (see [16]). A neutrosophic set (NS) $P$ in a finite set $X$ is denoted as

$$
P=\left\{\left\langle x, T_{P}(x), I_{P}(x), F_{P}(x)\right\rangle \mid x \in X\right\},
$$

where $T_{P}(x), I_{P}(x)$, and $F_{P}(x)$ are called the truth, the indeterminacy, and the falsity-membership functions, respectively. Moreover, $T_{P}(x), I_{P}(x)$ and $F_{P}(x)$ are the standard and nonstandard subsets of real numbers $] 0^{-}, 1^{+}[$ and satisfy

$$
0^{-} \leq \sup T_{P}(x)+\sup I_{P}(x)+\sup F_{P}(x) \leq 3^{+} .
$$

To extend the application of the NS in engineering and science areas, Ye [18] defined the simplified neutrosophic set (SNS).

Definition 2 (see [18]). A simplified neutrosophic set (SNS) $Q$ in a finite set $X$ is described in the following form:

$$
Q=\left\{\left\langle x, T_{Q}(x), I_{Q}(x), F_{Q}(x)\right\rangle \mid x \in X\right\},
$$

where $T_{\eta}(x), I_{\eta}(x)$, and $F_{\eta}(x)$ represent the truth, the indeterminacy, and the falsity-membership functions, respectively, and satisfy

$$
\begin{aligned}
& 0 \leq T_{Q}(x), I_{Q}(x), F_{Q}(x) \leq 1, \\
& 0 \leq T_{Q}(x)+I_{Q}(x)+F_{Q}(x) \leq 3 .
\end{aligned}
$$

For convenience, element $q=\left(T_{q}, I_{q}, F_{q}\right)$ is generally named as a simplified neutrosophic number (SNN), and the complement of $q=\left(T_{q}, I_{q}, F_{q}\right)$ is defined as $q^{c}=\left(F_{q}, 1-I_{q}, T_{q}\right)$.

Let $q=\left(T_{q}, I_{q}, F_{q}\right)$ and $s=\left(T_{s}, I_{s}, F_{s}\right)$ be two SNNs; some of mathematical operations are provided by Ye [18]:

(1) $q \oplus s=\left(T_{q}+T_{s}-T_{q} * T_{s}, I_{q} * T_{s}, F_{q} * F_{s}\right)$

(2) $\lambda q=\left(1-\left(1-T_{q}\right)^{\lambda},\left(I_{q}\right)^{\lambda},\left(F_{q}\right)^{\lambda}\right)(\lambda>0)$

Definition 3 (see [19]). Let $x_{i}=\left(T_{x_{i}}, I_{x_{i}}, F_{x_{i}}\right)(i=1,2)$ be two SNNs; then, the Hamming distance measure between $x_{1}$ and $x_{2}$ is presented as follows:

$$
d_{\mathrm{SNN}}\left(x_{1}, x_{2}\right)=\frac{1}{3}\left(\left|T_{x_{1}}-T_{x_{2}}\right|+\left|I_{x_{1}}-I_{x_{2}}\right|+\left|F_{x_{1}}-F_{x_{2}}\right|\right) \text {. }
$$

On the basis of the distance measure defined in equation (5), Sahin and Kucuk [47] proposed a SN similar measure between $x_{1}$ and $x_{2}$ as follows: 


$$
\operatorname{SM}_{\mathrm{SNN}}\left(x_{1}, x_{2}\right)=\frac{d_{\mathrm{SNN}}\left(x_{1}, x_{2}^{c}\right)}{d_{\mathrm{SNN}}\left(x_{1}, x_{2}\right)+d_{\mathrm{SNN}}\left(x_{1}, x_{2}^{c}\right)} .
$$

\subsection{The SNOWD Measure}

Definition 4 (see [47]). Let $Q=\left\{q_{1}, q_{2}, \ldots, q_{n}\right\}$ and $S=\left\{s_{1}, s_{2}, \ldots, s_{n}\right\}$ be two collections of SNNs, and $d_{\mathrm{SNN}}\left(q_{i}, s_{i}\right)$ is the distance between SNNs $q_{i}$ and $s_{i}$; then, the simplified neutrosophic weighted distance (SNWD) measure can be defined as follows:

$$
\operatorname{SNWD}(Q, S)=\left(\sum_{i=1}^{n} w_{i}\left(d_{\mathrm{SNN}}\left(q_{i}, s_{i}\right)\right)^{k}\right)^{1 / k},
$$

where $k>0$ and $\omega=\left(\omega_{1}, \omega_{2}, \ldots, \omega_{n}\right)$ is the weighted vector of $d_{\mathrm{SNN}}\left(q_{i}, s_{i}\right)$ such that $\omega_{i} \in[0,1]$ and $\sum_{i=1}^{n} \omega_{i}=1$.

Motivated by the OWD measure [31], Sahin and Kucuk [47] proposed the conception of the SNOWD measure, whose significance property is the ordered mechanism for the aggregated information.

Definition 5 (see [47]). Let $Q=\left\{q_{1}, q_{2}, \ldots, q_{n}\right\}$ and $S=\left\{s_{1}, s_{2}, \ldots, s_{n}\right\}$ be two sets of SNNs, and $d_{\mathrm{SNN}}\left(q_{i}, s_{i}\right)$ is the distance between SNNs $q_{i}$ and $s_{i}$; then, the simplified neutrosophic ordered weighted distance (SNOWD) measure is defined as

$$
\operatorname{SNOWD}(Q, S)=\left(\sum_{i=1}^{n} w_{i}\left(d_{\mathrm{SNN}}\left(q_{\sigma(i)}, s_{\sigma(i)}\right)\right)^{k}\right)^{1 / k},
$$

where $d_{\mathrm{SNN}}\left(q_{\sigma(i)}, s_{\sigma(i)}\right)(i=1,2, \ldots, n)$ is the reorder values such that $d_{\mathrm{SNN}}\left(q_{\sigma(1)}, s_{\sigma(1)}\right) \quad \geq d_{\mathrm{SNN}}\left(q_{\sigma(2)}, s_{\sigma(2)}\right) \geq \cdots \geq$ $d_{\mathrm{SNN}}\left(q_{\sigma(n)}, s_{\sigma(n)}\right)$. The associated weight vector of the SNOWD is $w=\left(w_{1}, w_{2}, \ldots, w_{n}\right)$ with $\sum_{i=1}^{n} w_{i}=1$ and $w_{i} \in[0,1]$.

The SNOWD measure possesses some good properties that the OWD also has, including boundedness, commutativity, idempotency, and monotonicity. However, the
SNOWD can only consider the weights of ordered deviations, but fail to reflect the weights (importance) of aggregated arguments that the SNWD can. Therefore, we shall propose an integrated weighted distance measure to eliminate the existing defects in the SNOWD measure.

\section{SN Integrated Weighted Distance (SNIWD) Measure}

It is observed from Definitions 1 and 5 that the SNWD can reflect the importance of the input argument but fails to account for the positions' weights of the ordered distances that the SNOWD can, while the SNOWD cannot emphasize the importance of aggregated deviations that the SNWD can. To solve the limitations, we present the SN integrated weighted distance (SNIWD) measure that can combine both merits of the SNOWD and the SNWD measures.

Definition 6. Let $Q=\left\{q_{1}, q_{2}, \ldots, q_{n}\right\}$ and $S=\left\{s_{1}, s_{2}, \ldots, s_{n}\right\}$ be two collections of SNNs, and $d_{\mathrm{SNN}}\left(q_{i}, s_{i}\right)$ is the distance between SNNs $q_{i}$ and $s_{i}$; then, the SNIWD measure is defined as

$$
\operatorname{SNIWD}(Q, S)=\left(\sum_{i=1}^{n} \psi_{i}\left(d_{\mathrm{SNN}}\left(q_{\sigma(i)}, s_{\sigma(i)}\right)\right)^{k}\right)^{1 / k},
$$

where the integrated weights $\psi_{i}$ are defined as

$$
\psi_{i}=\varepsilon w_{i}+(1-\varepsilon) \omega_{\sigma(i)},
$$

wherein $\omega_{i}$ is the weight of $d_{\mathrm{SNN}}\left(q_{i}, s_{i}\right)(i=1,2, \ldots, n)$ such that $\omega_{i} \in[0,1]$ and $\sum_{i=1}^{n} \omega_{i}=1, w_{i}$ is the relative weight of the SNIWD satisfying $\sum_{i=1}^{n} w_{i}=1$ and $w_{i} \in[0,1]$, and $\varepsilon$ is a real parameter satisfying $\varepsilon \in[0,1]$.

Obviously, when $\varepsilon=1$ and $\varepsilon=0$, the SNIWD is generalized to the SNOWD and the SNWD measures, respectively. Therefore, the SNIWD can be viewed as a combination of the SNOWD and SNWD measures, which can be proved by the following formula:

$$
\operatorname{SNIWD}(Q, S)=\left(\sum_{i=1}^{n} \psi_{i}\left(d_{\mathrm{SNN}}\left(q_{\sigma(i)}, s_{\sigma(i)}\right)\right)^{k}\right)^{1 / k}=\left(\varepsilon \sum_{i=1}^{n} w_{i}\left(d_{\mathrm{SNN}}\left(q_{\sigma(i)}, s_{\sigma(i)}\right)\right)^{k}+(1-\varepsilon) \sum_{i=1}^{n} \omega_{i}\left(d_{\mathrm{SNN}}\left(q_{i}, s_{i}\right)\right)^{k}\right)^{1 / k}
$$

Example 1. Let

$$
\begin{aligned}
Q & =\{(0.9,0.4,0.7),(0.6,0.2,0.4),(0.4,0.8,0.5),(0.7,0.1,0.6)\}, \\
S & =\{(0.5,0.5,0.3),(0.5,0.4,0.1),(0.3,0.7,0.4),(0.4,0.4,0.1)\},
\end{aligned}
$$

be two collections of SNNs; then, the computational procedure of the SNIWD is listed as follows:
(1) Utilize equation
(5) to calculate $d_{\mathrm{SNN}}\left(q_{i}, s_{i}\right)$ $(i=1,2, \ldots, 4)$ :

$$
\begin{aligned}
& d_{\mathrm{SNN}}\left(q_{1}, s_{1}\right)=0.3, \\
& d_{\mathrm{SNN}}\left(q_{2}, s_{2}\right)=0.2, \\
& d_{\mathrm{SNN}}\left(q_{3}, s_{3}\right)=0.1, \\
& d_{\mathrm{SNN}}\left(q_{4}, s_{4}\right)=0.4 .
\end{aligned}
$$

(2) Rank $d_{\mathrm{SNN}}\left(q_{i}, s_{i}\right) \quad(i=1,2, \ldots, 4)$ according to the decreasing order: 


$$
\begin{aligned}
& d_{\mathrm{SNN}}\left(q_{\sigma(1)}, s_{(1)}\right)=d_{\mathrm{SNN}}\left(q_{4}, s_{4}\right)=0.4 \\
& d_{\mathrm{SNN}}\left(q_{\sigma(2)}, s_{(2)}\right)=d_{\mathrm{SNN}}\left(q_{1}, s_{1}\right)=0.3 \\
& d_{\mathrm{SNN}}\left(q_{\sigma(3)}, s_{(3)}\right)=d_{\mathrm{SNN}}\left(q_{2}, s_{2}\right)=0.2 \\
& d_{\mathrm{SNN}}\left(q_{\sigma(3)}, s_{(4)}\right)=d_{\mathrm{SNN}}\left(q_{3}, s_{3}\right)=0.1
\end{aligned}
$$

(3) Let $w=(0.22,0.28,0.36,0.14)$ and $\omega=(0.2,0.4$, $0.1,0.3)$; then, compute the integrated weights $\psi_{i}$ according to equation (10) (let $\varepsilon=0.6$ ):

$$
\begin{aligned}
& \psi_{1}=0.6 \times 0.22+(1-0.6) \times 0.3=0.252, \\
& \psi_{2}=0.6 \times 0.28+(1-0.6) \times 0.2=0.248, \\
& \psi_{3}=0.6 \times 0.36+(1-0.6) \times 0.4=0.376, \\
& \widehat{w}_{4}=0.6 \times 0.14+(1-0.6) \times 0.1=0.124 .
\end{aligned}
$$

(4) Let $k=2$; then, calculate the distance between Qand Sutilizing the SNIWD measure defined in equation (9):

$$
\begin{aligned}
\operatorname{SNIWD}(Q, S) & =\left(\sum_{i=1}^{4} \psi_{i}\left(d_{\mathrm{SNN}}\left(q_{\sigma(i)}, s_{\sigma(i)}\right)\right)^{2}\right)^{1 / 2} \\
& =\left(0.252 \times 0.4^{2}+0.248 \times 0.3^{2}+0.376 \times 0.2^{2}+0.124 \times 0.1^{2}\right)^{1 / 2} \\
& =0.2809
\end{aligned}
$$

We can also illustrate the aggregation by applying the SNIWD measure given in equation (11):

$$
\begin{aligned}
\operatorname{SNIWD}(Q, S) & =\left(\sum_{i=1}^{4} \psi_{i}\left(d_{\mathrm{SNN}}\left(q_{\sigma(i)}, s_{\sigma(i)}\right)\right)^{2}\right)^{1 / 2} \\
& =\left(0.6 \times\left(\sum_{i=1}^{n} w_{i}\left(d_{\mathrm{SNN}}\left(q_{\sigma(i)}, s_{\sigma(i)}\right)\right)^{2}\right)+(1-0.6) \times\left(\sum_{i=1}^{n} \omega_{i}\left(d_{\mathrm{SNN}}\left(q_{i}, s_{i}\right)\right)^{2}\right)\right)^{1 / 2} \\
& =(0.6 \times 0.0762+(1-0.6) \times 0.083)^{1 / 2} \\
& =0.2809 .
\end{aligned}
$$

Obviously, the same results are rendered by both methods. Following the aforementioned definitions and the example, we can see that the SNIWD possesses the dual aggregated functions by combining the ordered weighted and arithmetic weighed methods, i.e., it covers both features of the previous SNOWD and the SNWD measures as it weights both the deviations and their ordered positions. Thus, it can not only reflect the weights of the input arguments themselves but also highlight the importance of their ordered positions during aggregation process. Moreover, it provides a possibility for decision makers to select suitable parameters according to actual demands or interests.

The SNIWD measure generalizes a wide range of SN distance measures by designing different values of the weights and parameters, for example:

Remark 1. Let $k=1$; then, we obtain the $\mathrm{SN}$ integrated weighted Hamming distance (SNIWHD) measure, and the $\mathrm{SN}$ integrated weighted Euclidean distance (SNIWED) measure is formed when $k=2$.
Remark 2. If $\varepsilon=1$, then the SNIWD is reduced to the SNOWD measure. Thus, all particular SN distance measures of the SNOWD mentioned in the result of Sahin and Kucuk [47] are the SNIWD's special cases, for example:

(i) The SN Hamming ordered weighted distance (SNHOWD) measure $(k=1)$

(ii) The SN Euclidean ordered weighted distance (SNEOWD) measure $(k=2)$

(iii) The SN geometric ordered weighted distance (SNGOWD) measure $(k \longrightarrow 0)$

(iv) Maximum $\mathrm{SN}$ distance measure $(w=(1,0,0, \ldots, 0))$

(v) Minimum SN distance measure $(w=(0,0, \ldots 0,1))$

(vi) Normalized $\mathrm{SN}$ distance measure $(w=(1 / n, 1 / n, \ldots, 1 / n))$

Remark 3. If $\varepsilon=0$, then the SNIWD is reduced to the SNWD measure. Then, we can achieve various families of the 
SNWD that can be seen as the SNIWD's particular status, such as:

(i) The SN Hamming weighted distance (SNHWD) measure $(k=1)$

(ii) The SN Euclidean weighted distance (SNEWD) measure $(k=2)$

(iii) The SN geometric weighted distance (SNGWD) measure $(k \longrightarrow 0)$

(iv) Normalized SN distance measure $(\omega=(1 / n, t 1 / n n, q \ldots h, 1 / n)$

Remark 4. By applying similar analysis introduced in the recent literature [48-53], more other cases of the SNIWD measure can be created, such as the the centered-SNIWD, median-SNIWD, and the Olympic-SNIWD measures.

The following theorems show that the SNIWD measure satisfies some desirable properties of monotonicity, boundedness, idempotency, commutativity, and reflexivity.

Theorem 1 (monotonicity). If $d_{\mathrm{SNN}}\left(q_{i}, s_{i}\right) \geq d_{\mathrm{SNN}}\left(q_{i}^{\prime}, s_{i}^{\prime}\right)$ for $i=1,2, \ldots, n$, then

$\operatorname{SNIWD}\left(\left(q_{1}, s_{1}\right), \ldots,\left(q_{n}, s_{n}\right)\right) \geq \operatorname{SNIWD}\left(\left(q_{1}^{\prime}, s_{1}^{\prime}\right), \ldots,\left(q_{n}^{\prime}, s_{n}^{\prime}\right)\right)$.

Theorem 2 (idempotency). If $d_{\mathrm{SNN}}\left(q_{i}, s_{i}\right)=d$ for $i=1,2, \ldots, n$, then

$$
\operatorname{SNIWD}\left(\left(q_{1}, s_{1}\right), \ldots,\left(q_{n}, s_{n}\right)\right)=d
$$

Theorem 3 (boundedness). Let $d_{\mathrm{min}}=\min _{i}\left(d_{\mathrm{SNN}}\left(q_{i}, s_{i}\right)\right)$ and $d_{\max }=\max _{i}\left(d_{\mathrm{SNN}}\left(q_{i}, s_{i}\right)\right)$; then,

$$
d_{\min } \leq \operatorname{SNIWD}\left(\left(q_{1}, s_{1}\right), \ldots,\left(q_{n}, s_{n}\right)\right) \leq d_{\max } .
$$

Theorem 4 (commutativity). If $\left(\left(q_{1}^{\prime}, s_{1}^{\prime}\right), \ldots,\left(q_{n}^{\prime}, s_{n}^{\prime}\right)\right)$ is any permutation of $\left(\left(q_{1}, s_{1}\right), \ldots,\left(q_{n}, s_{n}\right)\right)$, then

$$
\operatorname{SNIWD}\left(\left(q_{1}, s_{1}\right), \ldots,\left(q_{n}, s_{n}\right)\right)=\operatorname{SNIWD}\left(\left(q_{1}^{\prime}, s_{1}^{\prime}\right), \ldots,\left(q_{n}^{\prime}, s_{n}^{\prime}\right)\right) \text {. }
$$

Theorem 5 (reflexivity). If $q_{i}=s_{i}$ for $i=1,2, \ldots, n$, then

$$
\operatorname{SNIWD}\left(\left(q_{1}, s_{1}\right), \ldots,\left(q_{n}, s_{n}\right)\right)=0 \text {. }
$$

\section{Application in MAGDM}

As a generalization of various distance measures, the SNIWD is applicable to many fields, such as data analysis, decision-making, social management, pattern recognition, and financial investment. In this section, an application in the MAGDM problems is studied. Suppose that a MAGDM problem has $m$ different alternatives $B_{1}, B_{2}, \ldots, B_{m}$, and some experts $E_{1}, E_{2}, \ldots, E_{t}$ are consulted to assess $n$ finite attributes $C_{1}, C_{2}, \ldots, C_{n}$. Following the available information, the general procedure based on the SNIWD and entropy measures for MAGDM can be summarized as follows.

Step 1. Construct the $\mathrm{SN}$ individual decision matrix $R^{l}=\left(r_{i j}^{(l)}\right)_{m \times n}$, where $r_{i j}^{(l)}=\left(T_{i j}^{(l)}, I_{i j}^{(l)}, F_{i j}^{(l)}\right)$ provided by expert $e_{l}{ }^{i}(l=1,2, \ldots, t)$ is a SNN denoting the assessment of alternative $B_{i}$ with respect to attribute $C_{j}$.

Step 2. Determine the weight vector of experts (or decision makers) based on the similarity measure method [47]. In some actual problems, the weights of the experts cannot be determined beforehand. Thus, we introduce a method to derive the weights' information of experts based on the similar measures between individual opinions $R^{l}=\left(r_{i j}^{(l)}\right)_{m \times n}$ and the overall decision matrix $R^{*}=\left(r_{i j}^{(*)}\right)_{m \times n}$ :

$$
\operatorname{sm}\left(R^{(l)}, R^{*}\right)=\sum_{i=1}^{n} \sum_{j=1}^{m} \operatorname{sm}\left(r_{i j}^{(l)}, r_{i j}^{*}\right)
$$

where the distance measure $\operatorname{sm}\left(r_{i j}^{(l)}, r_{i j}^{*}\right)$ between $r_{i j}^{(l)}$ and $r_{i j}^{*}$ can be calculated by equation (6) and $r_{i j}^{(*)}=\left(T_{i j}^{(*)}, I_{i j}^{(*)}, F_{i j}^{(*)}\right)$ is the mean value of $r_{i j}^{(l)}=\left(T_{i j}^{(l)}, I_{i j}^{(l)}, F_{i j}^{(l)}\right)(l=1,2, \ldots, t)$ determined by the following formula:

$$
T_{i j}^{(*)}=\frac{1}{t} \sum_{l=1}^{t} T_{i j}^{(l)}, I_{i j}^{(*)}=\frac{1}{t} \sum_{l=1}^{t} I_{i j}^{(l)}, F_{i j}^{(*)}=\frac{1}{t} \sum_{l=1}^{t} F_{i j}^{(l)} .
$$

On the basis of the similar measures, the weight of expert $e_{l}(l=1,2, \ldots, t)$ can be derived by the following equation:

$$
\theta_{l}=\frac{\operatorname{sm}\left(R^{(l)}, R^{(*)}\right)}{\sum_{l=1}^{t} \operatorname{sm}\left(R^{(l)}, R^{(*)}\right)},
$$

where $\theta_{i} \in[0,1]$, and $\sum_{i=1}^{t} \theta_{i}=1$. Moreover, the weight of experts derived by this method has the desirable characteristic: the larger the similarity $\operatorname{sm}\left(R^{(l)}, R^{*}\right)$ is, the more closer the individual evaluation $R^{(l)}$ to the overall evaluation $R^{*}$ is and the larger the weight of expert $e_{l}(l=1,2, \ldots, t)$ is.

Step 3. Calculate the collective decision matrix $R=\left(\begin{array}{c}i j \\ r\end{array}\right)_{m \times n}$ using the SN weighted averaging (SNWA) operator [21], where $r_{i j}=\left(T_{i j}, I_{i j}, F_{i j}\right)=\sum_{l=1}^{t} \theta_{l} r_{i j}^{(l)}$.

Step 4. Determine the weight vector of the attribute. It is often difficult to express the weight information of the attribute in advance due to time limited or experts' professional knowledge. Thus, we develop an entropy-based method to derive the importance of attribute $C_{j}(j=1,2, \ldots, n)$ :

$$
\omega_{j}=\frac{1-E_{j}}{n-\sum_{j=1}^{n} E_{j}} .
$$

$\omega_{i} \in[0,1]$ and $\sum_{i=1}^{n} \omega_{i}=1$, and the entropy measure $E_{j}$ introduced by Biswas et al. [54] can be calculated from the following equationfd16:

$$
E_{j}=\frac{1}{m} \sum_{i=1}^{m}\left(1-\left|2 I_{i j}-1\right| \times\left(T_{i j}+F_{i j}\right)\right) .
$$


Step 5. Set ideal scheme $I=\left(I_{1}, I_{2}, \ldots, I_{n}\right)$ utilizing the following formula:

$$
I_{j}=\left(T_{I_{j}}, I_{I_{j}}, F_{I_{j}}\right)= \begin{cases}\left(\max _{i} T_{i j}, \min _{i} I_{i j}, \min _{i} F i_{j}\right), & \text { for the benefit attribute } \\ \left(\min _{i} T_{i j}, \max _{i} I_{i j}, \max _{i} F_{i j}\right), & \text { for the cost attribute. }\end{cases}
$$

Step 6. Apply the SNIWD measure to calculate the distances between alternative $B_{i}(i=1,2, \ldots, m)$ and ideal scheme $I$ :

$$
\operatorname{SWIND}\left(B_{i}, I\right)=\left(\sum_{j=1}^{m} \psi_{j} d_{\mathrm{SNN}}\left(r_{\sigma(i j)}, I_{\sigma(j)}\right)^{k}\right)^{1 / k}, \quad i=1,2, \ldots, n \text {. }
$$

Step 7. Rank the alternatives in accordance with the results obtained in the previous step, and hence, select the best choice.

\section{Numerical Case of Investment Selection}

In this section, we give a mathematical example of the investment selection problem [21] to verify the effectiveness and applicability of the presented method. A company would like to invest a sum of money to get a good return. Four possible alternatives are considered: (1) $B_{1}$ is a computer company; (2) $B_{2}$ is a food company; (3) $B_{3}$ is a car company; and (4) $B_{4}$ is an arms company. Three experts $\left\{E_{1}, E_{2}, E_{3}\right\}$ are invited to assess the companies from the following attributes: $C_{1}$ is the risk analysis; $C_{2}$ is the environmental impact analysis, and $C_{3}$ is the growth analysis, wherein $C_{1}$ and $C_{3}$ are of the benefit types, while $C_{2}$ belongs to the cost type. Then, the decision procedures are illustrated as follows.

Step 1. The individual SN decision matrix provided by experts is listed in Tables $1-3$.

Step 2. On the basis of the aforementioned decision matrix, the overall decision matrix $R^{*}=\left(r^{(*)}\right)_{m \times n}$ is calculated by using equation (24), listed in Table 4 .

Using equation (25), the similar measures between individual opinions $R^{(l)}(l=1,2,3)$ and the overall decision matrix $R^{*}$ are calculated as

$$
\begin{gathered}
\operatorname{sm}\left(R^{(1)}, R^{*}\right)=0.883, \\
\operatorname{sm}\left(R^{(2)}, R^{*}\right)=0.903, \\
\operatorname{sm}\left(R^{(3)}, R^{*}\right)=0.896 .
\end{gathered}
$$

Thus, the weights of experts are derived as

$$
\begin{aligned}
& \theta_{1}=0.329, \\
& \theta_{2}=0.337, \\
& \theta_{3}=0.334 .
\end{aligned}
$$

Step 3. According to the weights of the experts, the collective SN decision matrix can be calculated by using the SNWA operator, presented in Table 5.

Step 4. Applying equations (26) and (27), the weight vector of attributes is computed as $\omega=(0.3634,0.2862,0.3504)$.

Step 5. The results of the ideal scheme by applying equation (28) are calculated as given in Table 6.

Step 6. Without loss of generality, let the parameter and wight vector of the SNIWD measure be $\varepsilon=0.5$ and $w=(0.3,0.5,0.2)$, respectively. Then, based on the weights of attributes obtained in Step 5, the distances between each alternative $B_{i}(i=1,2,3,4)$ and ideal scheme $I$ are calculated by using equation (29):

$$
\begin{aligned}
& \operatorname{SNIWD}\left(B_{1}, I\right)=0.1526 \\
& \operatorname{SNIWD}\left(B_{2}, I\right)=0.0922 \\
& \operatorname{SNIWD}\left(B_{3}, I\right)=0.1070 \\
& \operatorname{SNIWD}\left(B_{4}, I\right)=0.0945
\end{aligned}
$$

Step 7. Rank all the alternatives in accordance with the decreasing values of $\operatorname{SNIWD}\left(B_{i}, I\right)$. The smaller the value of $\operatorname{SNIWD}\left(B_{i}, I\right)$, the closest $B_{i}$ to the ideal scheme, and thus the better alternative $B_{i}$. Therefore, the alternatives can be ranked as

$$
B_{2}>B_{4}>B_{3}>B_{1} \text {. }
$$

Hence, the best alternative is $B_{2}$.

Moreover, we can apply some special cases of the SNWID mentioned in Section 4 to calculate the relative distances from the alternatives to the ideal scheme for obtaining a more comprehensive picture. The aggregation results are shown in Table 7 , and the subsequent ranking order is listed in Table 8 .

It can be seen in Table 8 that different ranking lists can be achieved from different cases of the SNIWD measures. Therefore, this method presents a more flexible mechanism for decision makers to choose different schemes according to their own needs or actual situations.

To perform the applicability of the presented method, we conduct a comparative research on some existing approaches for handling SN decision-making problems. We select the correlation coefficient method proposed by Ye [55], cross-entropy method by Ye [56], TOSIS method developed by Zeng et al., [27], SNWA method introduced by 
TABLE 1: SN decision matrix $R^{(1)}$.

\begin{tabular}{lcrr}
\hline & $C_{1}$ & $C_{2}$ & $C_{3}$ \\
\hline$B_{1}$ & $(0.6,0.2,0.3)$ & $(0.5,0.1,0.2)$ & $(0.5,0.1,0.3)$ \\
$B_{2}$ & $(0.5,0.3,0.2)$ & $(0.5,0.3,0.3)$ & $(0.7,0.1,0.3)$ \\
$B_{3}$ & $(0.5,0.1,0.2)$ & $(0.3,0.1,0.3)$ & $(0.5,0.2,0.2)$ \\
$B_{4}$ & $(0.5,0.3,0.2)$ & $(0.7,0.2,0.2)$ & $(0.7,0.2,0.2)$ \\
\hline
\end{tabular}

TABLE 2: SN decision matrix $R^{(2)}$.

\begin{tabular}{lcrr}
\hline & $C_{1}$ & $C_{2}$ & $C_{3}$ \\
\hline$B_{1}$ & $(0.4,0.1,0.3)$ & $(0.6,0.2,0.2)$ & $(0.5,0.3,0.3)$ \\
$B_{2}$ & $(0.6,0.1,0.2)$ & $(0.5,0.2,0.3)$ & $(0.7,0.2,0.3)$ \\
$B_{3}$ & $(0.5,0.2,0.2)$ & $(0.3,0.2,0.4)$ & $(0.6,0.2,0.3)$ \\
$B_{4}$ & $(0.7,0.3,0.1)$ & $(0.5,0.1,0.2)$ & $(0.6,0.3,0.2)$ \\
\hline
\end{tabular}

TABLE 3: SN decision matrix $R^{(3)}$.

\begin{tabular}{lccr}
\hline & $C_{1}$ & $C_{2}$ & $C_{3}$ \\
\hline$B_{1}$ & $(0.3,0.2,0.3)$ & $(0.5,0.3,0.2)$ & $(0.5,0.2,0.3)$ \\
$B_{2}$ & $(0.6,0.1,0.2)$ & $(0.5,0.2,0.2)$ & $(0.6,0.1,0.2)$ \\
$B_{3}$ & $(0.4,0.2,0.3)$ & $(0.2,0.2,0.5)$ & $(0.4,0.2,0.3)$ \\
$B_{4}$ & $(0.7,0,0.1)$ & $(0.4,0.3,0.2)$ & $(0.6,0.1,0.2)$ \\
\hline
\end{tabular}

TABLe 4: Overall decision matrix $R^{*}$.

\begin{tabular}{lcrr}
\hline & $C_{1}$ & $C_{2}$ & $C_{3}$ \\
\hline$B_{1}$ & $(0.448,0.159,0.262)$ & $(0.552,0.182,0.200)$ & $(0.500,0.182,0.300)$ \\
$B_{2}$ & $(0.569,0.144,0.200)$ & $(0.500,0.229,0.262)$ & $(0.670,0.126,0.262)$ \\
$B_{3}$ & $(0.469,0.159,0.229)$ & $(0.268,0.159,0.391)$ & $(0.507,0.200,0.262)$ \\
$B_{4}$ & $(0.644,0.000,0.126)$ & $(0.552,0.182,0.200)$ & $(0.637,0.182,0.200)$ \\
\hline
\end{tabular}

TABLE 5: Collective SN decision matrix $R$.

\begin{tabular}{lcrr}
\hline & $C_{1}$ & $C_{2}$ & $C_{3}$ \\
\hline$B_{1}$ & $(0.447,0.158,0.263)$ & $(0.536,0.182,0.200)$ & $(0.500,0.182,0.300)$ \\
$B_{2}$ & $(0.570,0.144,0.200)$ & $(0.500,0.229,0.262)$ & $(0.670,0.126,0.262)$ \\
$B_{3}$ & $(0.469,0.159,0.229)$ & $(0.268,0.159,0.392)$ & $(0.507,0.200,0.263)$ \\
$B_{4}$ & $(0.645,0.000,0.126)$ & $(0.551,0.190,0.200)$ & $(0.636,0.182,0.200)$ \\
\hline
\end{tabular}

TABLE 6: Ideal scheme.

\begin{tabular}{|c|c|c|}
\hline$C_{1}$ & $\mathrm{C}_{2}$ & $C_{3}$ \\
\hline$I \quad(0.645,0.000,0.126)$ & $(0.268,0.229,0.392)$ & $(0.670,0.126,0.200)$ \\
\hline
\end{tabular}

Peng et al. [21], ordered weighted SN cosine similarity measure [26], and power aggregation model provided by Liu and Luo [25]. All the ranking lists are illustrated in Table 9.

It is noted from Table 9 that the best choice is either $B_{2}$ or $B_{4}$, and the ranking lists of all alternatives may vary depending on the decision method used. The main reasons can be summarized as follows:

(1) The proposed SNIWD and the entropy model can efficiently eliminate the large deviation opinions provided by experts through the ordered weighting mechanism, which widely exists in MAGDM problems.

(2) An entropy model is put forward to derive the unknown weights' information of attributes in this paper. By contrast, the weights of attributes are determined by decision makers (experts) in advance in the aforementioned methods. 
TABLe 7: Aggregated results rendered by particular cases of the SNIWD.

\begin{tabular}{cccccccc}
\hline & Maximum & Minimum & SNWHD & SNWED & SNOWHD & SNOWED & SNIWHD \\
\hline$B_{1}$ & 0.1643 & 0.1087 & 0.1462 & 0.1487 & 0.1546 & 0.1563 & 0.1504 \\
$B_{2}$ & 0.1207 & 0.0207 & 0.0773 & 0.0883 & 0.0892 & 0.0961 \\
$B_{3}$ & 0.146 & 0.0233 & 0.0948 & 0.1068 & 0.0985 & 0.1073 \\
$B_{4}$ & 0.1713 & 0.0000 & 0.0595 & 0.0933 & 0.0633 & 0.0962 & 0.0966 \\
\hline
\end{tabular}

TABLE 8: Ranking results obtained by particular cases of the SNIWD.

\begin{tabular}{ll}
\hline Method & \multicolumn{1}{c}{ Ranking } \\
\hline Maximum & $B_{2}>B_{3}>B_{1}>B_{4}$ \\
Minimum & $B_{4}>B_{1}>B_{2}>B_{3}$ \\
SNWHD & $B_{2}>B_{4}>B_{3}>B_{1}$ \\
SNWED & $B_{2}>B_{4}>B_{3}>B_{1}$ \\
SNOWHD & $B_{4}>B_{2}>B_{3}>B_{1}$ \\
SNOWED & $B_{2}>B_{4}>B_{3}>B_{1}$ \\
SNIWHD & $B_{4}>B_{2}>B_{3}>B_{1}$ \\
\hline
\end{tabular}

TABLE 9: Ranking results obtained by the existing decision-making method.

\begin{tabular}{ll}
\hline Method & \multicolumn{1}{c}{ Ranking } \\
\hline Correlation coefficient method [55] & $B_{4}>B_{1}>B_{2}>B_{3}$ \\
Cross-entropy method [56] & $B_{4}>B_{2}>B_{3}>B_{1}$ \\
TOSIS method [27] & $B_{2}>B_{4}>B_{1}>B_{3}$ \\
SNWA method [21] & $B_{2}>B_{4}>B_{3}>B_{1}$ \\
Ordered weighted cosine similarity measure & $B_{2}>B_{4}>B_{3}>B_{1}$ \\
method [26] & $B_{4}>B_{2}>B_{3}>B_{1}$ \\
Power aggregation method [25] & \\
\hline
\end{tabular}

\section{Conclusions}

In this study, we present a new approach based on the integrated distance measure and entropy weights for MAGDM with SN information. The SNIWD measure is proposed to improve the defects of the previous methods. The main advantage of the SNIWD is that it combines the ordered weighted and arithmetic weighted functions for reflecting the SN deviations. Moreover, it generalizes a great many of SN distance measures, including the SNOWD and the SNWD. Then, we develop a MAGDM approach based on the SNIWD and the entropy method within SN situations, wherein the entropy measure is utilized to determine the unknown weight information. A case study regarding selection of a suitable investment case is given to illustrate the efficiency of the proposed framework. The results and comparative study with other existing models test the advantages and effectiveness of our method. The preponderances of the proposed method based on the SNIWD measure and the entropy weight are summed up as follows: (1) the existing approaches based on the ordered weighted distance measures in decision-making areas only pay attention to the weights of the ordered deviation. They fail to account for the importance of attributes. By contrast, the introduced method based on the SNIWD can effectively fuse both importance of the ordered deviations and attributes; (2) the attribute weight is given by decision makers in advance in the existing literature. However, we present an entropy measure method to derive the unknown attribute weight information, which helps to achieve a more objective result; and (3) the proposed method based on the SNIWD is more flexible as it provides a chance for the decision maker to select the appropriate parameters that are near to his or her interests or the needs of the decision-making problems.

In our subsequent study, we will consider some other applications of the proposed approach, such as education evaluation and social network. Some new extensions by using other variables are also considered in complex situations.

\section{Data Availability}

No data were used to support this study.

\section{Conflicts of Interest}

The authors declare no conflicts of interest.

\section{Acknowledgments}

This paper was supported by Major Humanities and Social Sciences Research Projects in Zhejiang Universities (no. 2018QN058), the Zhejiang Natural Science Foundation (Project no. LY20G030006), and the Ningbo Natural Science Foundation (no. 2019A610037).

\section{References}

[1] L. A. Zadeh, "Fuzzy sets," Information and Control, vol. 8, no. 3, pp. 338-353, 1965.

[2] K. T. Atanassov, "Intuitionistic fuzzy sets," Fuzzy Sets and Systems, vol. 20, no. 1, pp. 87-96, 1986.

[3] R. R. Yager, "Pythagorean membership grades in multicriteria decision making," IEEE Transactions on Fuzzy Systems, vol. 22, no. 4, pp. 958-965, 2014.

[4] T.-Y. Chen, "Remoteness index-based Pythagorean fuzzy VIKOR methods with a generalized distance measure for multiple criteria decision analysis," Information Fusion, vol. 41, pp. 129-150, 2018.

[5] H. Garg, "New logarithmic operational laws and their aggregation operators for Pythagorean fuzzy set and their applications," International Journal of Intelligent Systems, vol. 34, pp. 82-106, 2019.

[6] D. Liang, Z. Xu, D. Liu, and Y. Wu, "Method for three-way decisions using ideal TOPSIS solutions at Pythagorean fuzzy information," Information Sciences, vol. 435, pp. 282-295, 2018.

[7] X. Peng and G. Selvachandran, "Pythagorean fuzzy set: state of the art and future directions," Artificial Intelligence Review, vol. 52, no. 3, pp. 1873-1927, 2019. 
[8] D. Yu, Z. Xu, and W. Wang, "Bibliometric analysis of fuzzy theory research in China: a 30-year perspective," KnowledgeBased Systems, vol. 141, pp. 188-199, 2018.

[9] Z. Yang, T. Ouyang, X. Fu, and X. Peng, “A decision-making algorithm for online shopping using deep-learning-based opinion pairs mining and q -rung orthopair fuzzy interaction Heronian mean operators," International Journal of Intelligent Systems, vol. 35, no. 5, pp. 783-825, 2020.

[10] Z. L. Peng, H. Garg, J. Li, G. Srivastavad, and Z. Cao, "Investigation of multiple heterogeneous relationships using a q-rung orthopair fuzzy multi-criteria decision algorithm," Neural Computing and Applications, 2020.

[11] G. W. Wei and M. Lu, "Pythagorean fuzzy power aggregation operators in multiple attribute decision making," International Journal of Intelligent Systems, vol. 33, pp. 169-186, 2018.

[12] S. Zeng, Z. Mu, and T. Baležentis, "A novel aggregation method for pythagorean fuzzy multiple attribute group decision making," International Journal of Intelligent Systems, vol. 33, no. 3, pp. 573-585, 2018.

[13] S. Zeng, X. Peng, T. Baležentis, and D. Streimikiene, "Prioritization of low-carbon suppliers based on Pythagorean fuzzy group decision making with self-confidence level," Economic Research-Ekonomska Istraživanja, vol. 32, no. 1, pp. 1073-1087, 2019.

[14] C. Zhang, C. Chen, D. Streimikiene, and T. Balezentis, "Intuitionistic fuzzy MULTIMOORA approach for multicriteria assessment of the energy storage technologies," Applied Soft Computing, vol. 79, pp. 410-423, 2019.

[15] X. Zhang and Z. Xu, "Extension of TOPSIS to multiple criteria decision making with pythagorean fuzzy sets," International Journal of Intelligent Systems, vol. 29, no. 12, pp. 1061-1078, 2014.

[16] F. Smarandache, A Unifying Field in Logics. Neutrosophy: Neutrosophic Probability, Set and Logic, American Research Press, Rehoboth, MA, USA, 1999.

[17] H. Wang, F. Smarandache, Y. Q. Zhang, and R. Sunderraman, "Single valued neutrosophic sets," Multispace Multistruct, vol. 4, pp. 410-413, 2010.

[18] J. Ye, "A multicriteria decision-making method using aggregation operators for simplified neutrosophic sets," Journal of Intelligent \& Fuzzy Systems, vol. 26, no. 5, pp. 2459-2466, 2014a.

[19] J. Ye, "An extended TOPSIS method for multiple attribute group decision making based on single valued neutrosophic linguistic numbers," Journal of Intelligent \& Fuzzy Systems, vol. 28, no. 1, pp. 247-255, 2015.

[20] J.-j. Peng, J.-q. Wang, H.-y. Zhang, and X.-h. Chen, "An outranking approach for multi-criteria decision-making problems with simplified neutrosophic sets," Applied Soft Computing, vol. 25, pp. 336-346, 2014.

[21] J.-j. Peng, J.-q. Wang, J. Wang, H.-y. Zhang, and X.-h. Chen, "Simplified neutrosophic sets and their applications in multicriteria group decision-making problems," International Journal of Systems Science, vol. 47, no. 10, pp. 2342-2358, 2015.

[22] G. D. Küçük and R. Şahin, “A novel hybrid approach for simplified neutrosophic decision-making with completely unknown weight information," International Journal for Uncertainty Quantification, vol. 8, no. 2, pp. 161-173, 2018.

[23] J. Ye, "A netting method for clustering-simplified neutrosophic information," Soft Computing, vol. 21, no. 24, pp. 7571-7577, 2017.

[24] R. Sahin and P. D. Liu, "Possibility-induced simplified neutrosophic aggregation operators and their application to multi-criteria group decision-making," Journal of Experimental \& Theoretical Artificial Intelligence, vol. 4, pp. 769785, 2017.

[25] C. Liu and Y. Luo, "Power aggregation operators of simplified neutrosophic sets and their use in multi-attribute group decision making," IEEE/CAA Journal of Automatica Sinica, vol. 6, no. 2, pp. 575-583, 2019.

[26] J. Ye, "Generalized ordered weighted simplified neutrosophic cosine similarity measure for multiple attribute group decision making," International Journal of Cognitive Informatics and Natural Intelligence, vol. 14, no. 1, pp. 51-62, 2020.

[27] S. Zeng, D. Luo, C. Zhang, and X. Li, "A correlation-based TOPSIS method for multiple attribute decision making with single-valued neutrosophic information," International Journal of Information Technology \& Decision Making, vol. 19, no. 01, pp. 343-358, 2020.

[28] X. D. Peng and J. G. Dai, "A bibliometric analysis of neutrosophic set: two decades review from 1998 to 2017," Artificial Intelligence Review, vol. 53, pp. 199-255, 2020.

[29] A. Kaufmann, Introduction to the Theory of Fuzzy Subsets, Academic Press, New York, NY. USA, 1975.

[30] R. R. Yager, "On ordered weighted averaging aggregation operators in multicriteria decisionmaking," IEEE Transactions on Systems, Man, and Cybernetics, vol. 18, no. 1, pp. 183-190, 1988.

[31] Z. S. Xu and J. Chen, "An overview of distance and similarity measures of intuitionistic fuzzy sets," International Journal of Uncertainty, Fuzziness and Knowledge-Based Systems, vol. 16, no. 04, pp. 529-555, 2008.

[32] J. M. Merigó and A. M. Gil-Lafuente, "New decision-making techniques and their application in the selection of financial products," Information Sciences, vol. 180, no. 11, pp. 2085-2094, 2010.

[33] Z. Xu and M. Xia, "Distance and similarity measures for hesitant fuzzy sets," Information Sciences, vol. 181, no. 11, pp. 2128-2138, 2011.

[34] S. Zeng and W. Su, "Intuitionistic fuzzy ordered weighted distance operator," Knowledge-Based Systems, vol. 24, no. 8, pp. 1224-1232, 2011.

[35] S. Zeng, "Some intuitionistic fuzzy weighted distance measures and their application to group decision making," Group Decision and Negotiation, vol. 22, no. 2, pp. 281-298, 2013.

[36] M. Shakeel, S. Abdullah, and R. Ahmed, "Cubic ordered weighted distance operator and application in group decisionmaking," Journal of Intelligent Systems, vol. 29, no. 1, pp. 440-458, 2020.

[37] L. Zhou, H. Chen, and J. Liu, "Continuous ordered weighted distance measure and its application to multiple attribute group decision making," Group Decision and Negotiation, vol. 22, no. 4, pp. 739-758, 2013.

[38] L. Zhou, J. Wu, and H. Chen, "Linguistic continuous ordered weighted distance measure and its application to multiple attributes group decision making," Applied Soft Computing, vol. 25, pp. 266-276, 2014.

[39] V. G. Alfaro-García, J. M. Merigó, A. M. Gil-Lafuente, and J. Kacprzyk, "Logarithmic aggregation operators and distance measures," International Journal of Intelligent Systems, vol. 33, no. 7, pp. 1488-1506, 2018.

[40] V. G. Alfaro-Garcia, J. M. Merigo, L. Plata-Perez, G. G. Alfaro-Calderon, and A. M. Gil-Lafuente, "Induced and logarithmic distances with multi-region aggregation operators," Technological and Economic Development of Economy, vol. 25 , pp. $664-692,2019$.

[41] J. M. Merigó and M. Casanovas, "Decision-making with distance measures and induced aggregation operators," 
Computers \& Industrial Engineering, vol. 60, no. 1, pp. 66-76, 2011.

[42] W. Xue, S. Xian, and Y. Dong, "A novel intuitionistic fuzzy induced ordered weighted euclidean distance operator and its application for group decision making," International Journal of Intelligent Systems, vol. 32, no. 7, pp. 739-753, 2017.

[43] L. Yu, S. Zeng, J. M. Merigó, and C. Zhang, "A new distance measure based on the weighted induced method and its application to Pythagorean fuzzy multiple attribute group decision making," International Journal of Intelligent Systems, vol. 34, no. 7, pp. 1440-1454, 2019.

[44] C. Cao, S. Zeng, and D. Luo, "A single-valued neutrosophic linguistic combined weighted distance measure and its application in multiple-attribute group decision-making," Symmetry, vol. 11, no. 2, p. 275, 2019.

[45] J. M. Merigó, D. Palacios-Marqués, and P. Soto-Acosta, "Distance measures, weighted averages, OWA operators and Bonferroni means," Applied Soft Computing, vol. 50, pp. 356-366, 2017.

[46] S. Zeng and Y. Xiao, "A method based on TOPSIS and distance measures for hesitant fuzzy multiple attribute decision making," Technological and Economic Development of Economy, vol. 24, no. 3, pp. 969-983, 2018.

[47] R. Sahin and G. D. Kucuk, "Group decision making with simplified neutrosophic ordered weighted distance operator," Mathematical Methods in the Applied Sciences, vol. 41, pp. 4795-4809, 2018.

[48] T. Balezentis, D. Streimikiene, R. Melnikiene, and S. Z. Zeng, "Prospects of green growth in the electricity sector in Baltic States: pinch analysis based on ecological footprint," Resources Conservation and Recycling, vol. 142, pp. 37-48, 2019.

[49] S. D. Xian, Y. Xiao, L. Li, and D. X. Yu, "Trapezoidal Pythagorean fuzzy linguistic entropic combined ordered weighted Minkowski distance operator based on preference relations," International Journal of Intelligent Systems, vol. 34, pp. 2196-2224, 2019.

[50] Z. Yang, X. Li, H. Garg, and M. Qi, "Decision support algorithm for selecting an antivirus mask over COVID-19 pandemic under spherical normal fuzzy environment," International Journal of Environmental Research and Public Health, vol. 17, no. 10 , p. 3407,2020 c.

[51] Z. Yang and J. Chang, "Interval-valued pythagorean normal fuzzy information aggregation operators for multi-attribute decision making," IEEE Access, vol. 8, pp. 51295-51314, 2020d.

[52] D. Yu and $\mathrm{Z}$. Xu, "Intuitionistic fuzzy two-sided matching model and its application to personnel-position matching problems," Journal of the Operational Research Society, vol. 71, no. 2, pp. 312-321, 2020.

[53] S. Zeng, J. Chen, and X. Li, "A hybrid method for pythagorean fuzzy multiple-criteria decision making," International Journal of Information Technology \& Decision Making, vol. 15, no. 02, pp. 403-422, 2016.

[54] P. Biswas, S. Pramanik, and B. C. Giri, "Entropy based grey relational analysis method for multi-attribute decision making under single valued neutrosophic assessments," Neutrosophic Sets and Systems, vol. 2, pp. 102-110, 2014.

[55] J. Ye, "Multicriteria decision-making method using the correlation coefficient under single-valued neutrosophic environment," International Journal of General Systems, vol. 42, no. 4, pp. 386-394, 2013.

[56] J. Ye, "Single valued neutrosophic cross-entropy for multicriteria decision making problems," Applied Mathematical Modelling, vol. 38, no. 3, pp. 1170-1175, 2014 b. 